Cómo citar este artículo en Chicago: Díez Fischer, Francisco. "El desvío reflexivo de la cuestión de Dios en la memoria de juventud de Paul Ricœur”. Escritos 28, no. 61 (2020): 1-17. doi: http://dx.doi.org/10.18566/escr.v28n61.a01

\title{
El desvío reflexivo de la cuestión de Dios en la memoria de juventud de Paul Ricœur
}

\author{
The Reflexive Detour in the Issue of God within Paul Ricoeur's Memoirs of Youth
}

\author{
Francisco Díez Fischer ${ }^{1}$
}

\begin{abstract}
RESUMEN
En los años noventa, Paul Ricœur caracterizó su postura en torno a la cuestión metafísica de Dios como un agnosticismo filosófico. Consideró que esa característica era la marca de toda su obra. Sin embargo, en su memoria de juventud, escrita a los 20 años, Ricœur desarrolló una filosofía que nombraba a Dios y lo convertía en cuestión de un esfuerzo metafísico. Esa memoria permaneció inédita hasta 2017. Frente al reconocimiento de su agnosticismo filosófico, esta publicación genera preguntas ineludibles. Si el joven Ricœur se interesó por la aplicación del método de la filosofía reflexiva al problema de Dios ¿por qué justo este primer texto permaneció inédito?, ¿no fue publicado por contradecir la marca de la totalidad de su obra? El objetivo del presente estudio es esclarecer este origen que parece extraño a su descripción filosófica. Se examinarán tres puntos centrales de su memoria: $1^{\circ}$ ) el credo filosófico establecido en el inicio de su trabajo, $2^{\circ}$ ) las decisiones asumidas en su desarrollo, y $3^{\circ}$ ) la estructura de una conclusión imposible con la que finaliza.
\end{abstract}

Palabras clave: Filosofía reflexiva; Paul Ricœur; Dios; Metafísica; Agnosticismo; Agnosticismo filosófico.

\section{ABSTRACT}

During the 90s of the twentieth century, Paul Ricoeur designated his view concerning the metaphysical issue of God as philosophical agnosticism. He thought that feature to be the hallmark of his entire work. However, in his Memoirs of Youth [Méthode réflexive appliquée au problème de Dieu chez Lachelier et Lagneau], which he wrote during his twenties, Ricouer developed a philosophy that named God and that made it a metaphysical issue. This memoir remained unpublished until 2017. Faced with his self-proclaimed philosophical agnosticism, this work raises some questions. If the early Ricoeur was interested in applying the method of reflexive philosophy to the issue of God, why this early work remained unpublished? Was it published just because it contradicts the hallmark of his entire work? The purpose of the article is to shed light on the origin of this memoir, which seems strange due to his own description of his philosophical work. Therefore, it addresses three issues within the memoir: the philosophical ideas established in the beginning, the decisions he took during its development, and the structure of an impossible conclusion which ends it.

Keywords: Reflexive Philosophy; Paul Ricoeur; God; Metaphysics; Agnosticism; Philosophical Agnosticism.

1 Doctor en Filosofía por la Universidad de Buenos Aires. Docente de Antropología Filosófica en la Facultad de Filosofía y Letras de la Universidad Católica Argentina. Investigador Adjunto del CONICET, Argentina.

Correo electrónico: franciscodiez@uca.edu.ar 


\section{Introducción: planteamiento del problema}

$\mathrm{H}$

ay una máxima que dice: “Todo gran filósofo tiene detrás una metafísica”2, y con ella una idea de Dios -el famoso Dios de los filósofos que no tiene templos, ni creyentes que le recen-. En el caso de Paul Ricœur, la aplicación de esta máxima no es tarea sencilla por dos razones: primero, porque hermenéutica y metafísica son, en general, dos dimensiones de la filosofía que no suelen considerarse compatibles. Por el contrario, se presume que la hermenéutica conlleva una crítica a la metafísica onto-teológica como etapa superada de la filosofía. Segundo, porque en sus obras de madurez, Ricœur predicó expresamente un agnosticismo filosófico respecto a la cuestión metafísica de Dios que "le prohibió elaborar una teología filosófica en buena y debida forma, lo que no le impidió interesarse en el potencial de inteligibilidad de la exégesis bíblica"'3. Al final de la introducción de Soi-même comme un autre (1990), Ricœur formula su credo agnóstico diciendo:

(...) pienso no haber ofrecido a mis lectores más que argumentos que no comprometen la posición del lector, sea ésta de rechazo, de aceptación o dejada en suspenso, respecto a la fe bíblica. Se observará que este ascetismo del argumento que, creo, marca toda mi obra filosófica, conduce a un tipo de filosofía de la que está ausente la nominación efectiva de Dios y en la que el problema mismo de Dios, como cuestión filosófica, se mantiene en un aplazamiento (suspens), que se podría llamar agnóstico, como ponen de manifiesto las últimas líneas del estudio décimo. Para no hacer excepción a este aplazamiento (suspens), la única prolongación dada a los nueve estudios que competen expresamente a una fenomenología hermenéutica, consiste en una investigación ontológica que no da motivo a ninguna amalgama ontoteológica. $^{4}$

El tono post-metafísico y la clave heideggeriana de una crítica a la metafísica como onto-teología son evidentes y se resumen en lo que se podría llamar la epoché de la cuestión metafísica de Dios en su posición filosófica. El problema -que motiva este trabajo- es que esta posición, que, según él mismo cree, marca toda su obra filosófica, no ha sido siempre una postura sostenida. No tanto porque durante los años noventa Ricœur se embarcara en una aventura por las fronteras de la filosofía en vistas a desarrollar

2 Frase pronunciada por Jean Grondin en su conferencia “¿En qué consiste el sentido hermenéutico?”, en el VII Seminario de Fenomenología existencia: Sensibilidad y hermenéutica tras una ética de la solicitud, realizado el 23 de agosto de 2018 en la Universidad de la Frontera, Temuco, Chile.

3 Jean Greisch, "Préface" a Méthode réflexive appliquée au problème de Dieu chez Lachelier et Lagneau, (Philosophie \& théologie) (Paris: Editions du Cerf. Edición de Kindle, 2017), 9. Algunos de los escritos de Ricœur sobre exégesis bíblica son: "Exégesis y hermenéutica" (1971); "Hermenéutica del testimonio" (1972); "Manifestación y proclama" (1974); "La filosofía de la especificidad del lenguaje religioso" (1975); "Hermenéutica de la idea de Revelación" (1977); "Nombrar a Dios" (1977), "Pensar la Biblia" (1998); "La hermeneútica bíblica" (2001); entre muchos otros.

4 Paul Ricœur, Soi-même comme un autre (Paris: Ed. Du Seuil, 1990) XXXVIII. Si bien se ha decidido mantener la traducción de suspens por aplazamiento que utiliza la edición castellana, la idea misma de suspens refiere más bien a la operación suspensiva o a la interrupción de un camino que no es retomado más adelante. Respecto al final del estudio $10^{\circ}$ al que refiere, frente a la conminación levinasiana del Otro, afirma: "Quizá el filósofo, en cuanto filósofo, debe confesar que no sabe y no puede decir si este Otro, fuente de la conminación, es un otro al que yo pueda contemplar o que pueda mirarme, o son mis antepasados de los que no existe representación - tan constitutiva de mí mismo es mi deuda respecto a ellos-, o Dios - Dios vivo, Dios ausente-, o un lugar vacío. En esta aporía del Otro, el discurso filosófico se detiene." Ricœur, Soi-même comme un autre, 397. 
una hermenéutica y una fenomenología de la religión ${ }^{5}$, sino, sobre todo, porque, en sus comienzos, hizo una filosofía que nombraba efectivamente a Dios y lo convertía en cuestión central de un esfuerzo metafísico. La pretensión de este inicio fue el origen-fuente de su filosofar y se hizo texto en su primer escrito filósofo, titulado Méthode réflexive appliquée au problème de Dieu chez Lachelier et Lagneau. Se trata de la memoria para la obtención del Diploma de Estudios Superiores en la Universidad de Rennes bajo la dirección de Léon Brunschvicg; redactada entre 1933-1934, cuando Ricœur tenía sólo 20 años, y que permaneció inédita hasta su publicación en el año 2017. En sí mismo, el texto tiene el valor de ser el primer escrito filosófico de juventud de un gran filósofo que en aquellos años era un "militante activo" de la Federación de Estudiantes Cristianos. ${ }^{6}$ No obstante, en perspectiva del agnosticismo filosófico pregonado por el Ricœur maduro, la memoria motiva interrogantes ineludibles en relación con su tema. Si el joven Ricœur se interesó por la aplicación al problema de Dios del método de la filosofía reflexiva -su tradición de origen según él mismo reconoce ${ }^{7}-¿$ por qué justo este primer texto permaneció inédito?, ¿no fue hecho público por contradecir la marca expresa de su obra? En tanto fue su inicio filosófico, ¿no es evidente que debía ser considerado parte de la totalidad de su labor? Las respuestas podrían concretarse en dos direcciones. $1^{\circ}$ ) Ricœur puede haber considerado que una memoria de juventud sobre autores tan olvidados durante el siglo XX, como Jules Lachelier (1832-1918) y Jules Lagneau (1851-1894), no tenía el valor filosófico suficiente para ser publicada. Contra esta lectura, varios de sus intérpretes (Greisch, Capelle, Vallée, Grondin) consideran que, a pesar del carácter demodé de los pensadores examinados, en esta obra temprana se llega a ver, en estado germinal, muchas de las ideas que Ricœur desarrolló luego en sus obras más conocidas: la necesidad del rodeo reflexivo, el método crítico de confrontación, el primado de la acción sobre la especulación, la importancia de la persona, la inseparabilidad de reflexión y vida, el problema del mal, etc. ${ }^{8}$ Este valor como proto-fuente inicial para comprender el origen y evolución de la filosofía ricœuriana hace difícil menospreciar o relativizar la valía filosófica de esta obra. $2^{\circ}$ ) En tanto su posición de madurez fue la de un expreso agnosticismo filosófico, un escrito dedicado de forma tan manifiesta a la cuestión metafísica de Dios le representaría, sin lugar a duda, algo problemático. Al contradecir expresamente la posición que creyó sostener como marca de toda su obra, se puede pensar que fue un texto deliberadamente inédito y oculto. En ese caso, su publicación descubre hoy que, en el inicio de la filosofía de Ricœur, se anudaría una primera paradoja

5 Cf. Paul Ricœur, Lectures III, Aux frontières de la philosophie (Paris: Ed. Le Seuil, 1994).

6 En esos años, Ricœur ensayaba conjugar su doble pertenencia al socialismo y al protestantismo tanto en su manera de escribir como de obrar. Cf. François Dosse, Paul Ricœur: Les sens d'une vie (1913-2005), Edition revue et augmenté (Paris: La Découverte/ Poche, 2001, 2008), 47-48.

7 La tradición francesa de la filosofía reflexiva fue iniciada por Maine de Biran, y prolongada por Ravaisson, Boutroux, Lachelier, Lagneau, Brunschvicg y Nabert. Se elaboró sobre la herencia cartesiana, espinosista pero también en diálogo crítico con Fichte, Kant y Hegel. Como el mismo Ricœur reconoce, gracias al paso por Lachelier y Lagneau "me encontré iniciado y de hecho incorporado a la tradición de la filosofía reflexiva francesa, pariente del neokantismo alemán.” Paul Ricœur, Autobiografía intelectual (Buenos Aires: Ed. Nueva Visión, 2007), 17.

8 A pesar de dejarse leer con la familiaridad de las ideas germinales del Ricœur maduro, esta obra no sólo se lee con extrañeza porque su autor no hable de hermenéutica, ni de fenomenología, ni cite a quienes más tarde serán sus referentes fundamentales (Marcel, Jaspers, Husserl, Heidegger, Nabert). Creo que hay otras dos extrañezas. Por un lado, para quien conoce y está familiarizado con el lenguaje de la filosofía ricœuriana, sin absolutos, suena extraño leer tan expresamente sobre Dios. Por eso, este trabajo será pródigo en citas para dejar ver cómo piensa y escribe ese extraño Ricœur de juventud. Por otro, como el mismo Ricœur maduro reconoce, cada uno de sus libros y escritos han surgido como una totalidad a partir de los temas fragmentarios y aporéticos que han quedado pendientes en escritos y libros precedentes. Esta obra tiene la dificultad de ser el primer escrito de Ricoeur, es decir, un escrito que pretende ser una primera totalidad, sin precedentes, que abre problemas para todas las obras venideras. 
fundamental que recorrería la verdadera totalidad de su obra: la asunción primera y el aplazamiento posterior de la cuestión metafísica de Dios.

En perspectiva de esta segunda respuesta, se puede considerar que "la elección de un tema de investigación tiene siempre algo que ver con las interrogaciones que son más íntimas y afloran muy tempranamente en un verdadero pensador." Asumiendo la validez de este supuesto, el carácter excepcional del texto, es decir, que sea el primer escrito filosófico de Ricœur y se ocupe de la cuestión metafísica de Dios, creo que incrementa su valor, al menos en dos razones, para esclarecer el inicio de esta primera paradoja que movilizaría su obra: 1) Es un trabajo que muestra mejor que ningún otro el enraizamiento del pensamiento ricœuriano en la tradición de la filosofía reflexiva francesa, pero, al mismo tiempo, pone en evidencia que el método reflexivo con el que se compromete guarda un doble vínculo íntimo e inseparable: a) con la idea de Dios y de su existencia, examinado como cuestión metafísica directriz por la vía de la inmanencia, y b) con la necesaria finitud de una teología filosófica, entendida como fracaso de una metafísica sin trascendencia, sin distancia entre Dios y los hombres, que marca el lugar y da paso a la moral como Alternativa. 2) A esta cuestión de Dios que se encuentra en las fronteras del método reflexivo y de cualquier intento filosófico, el joven Ricœur la considera, por eso mismo, su punto de culminación. Se trata del origen, fin último y límite de un esfuerzo por alcanzar a Dios recorriendo la totalidad de las obras divinas:

Es necesario -escribe hacia el final de su memoria- rendir homenaje a Lachelier y a Lagneau por haber recordado que no hay más que un solo problema: el problema de Dios. Todo problema parcial no es más que arbitrariamente extraído de la totalidad del problema de Dios. Todo está en todo. Desde entonces, el punto de partida normal de una búsqueda de Dios será un inventario de todas sus posesiones. Ésta es la idea central del método reflexivo. ${ }^{10}$

¿Cómo podría resistirse a la tentación de ver aquí el escrito teológico de juventud de Ricœur en el sentido que Dilthey Habla de los escritos teológicos de juventud de Hegel, y Gadamer del escrito teológico de juventud de Heidegger? ${ }^{11}$ Si todo gran filósofo tiene detrás una metafísica, ¿la totalidad de la obra de Ricœur no comenzaría y tendría su origen (Urquelle) en esa culminación anticipada y nunca alcanzada; en ese "inacabamiento" como intención común a todas sus obras, que obliga a fracasar a cada una como totalidad?

A la luz de estas razones, se deja vislumbrar por qué la paradójica cuestión metafísica de Dios, en la filosofía de Ricœur, ha motivado la interpretación de que existe un "camino metafísico oculto o silencioso", como sostiene Grondin, o de "origen espiritualista", como afirma Vieillard-Baron, que se inicia en la memoria y se mantendría a lo largo de toda su obra. ${ }^{12}$ Se trataría de una búsqueda encubierta de Dios, de una

9 Jean Grondin, "Paul Ricceur, Méthode réflexive appliquée au problème de Dieu chez Lachelier et Lagneau", Revue des sciences religieuses [En línea], 92, n.1 (2018): 121-127, puesto en línea 01 enero 2019, consultado el 12 julio de 2019. URL: http://journals.openedition.org/rsr/4409, p. 2.

10 Ricœur, Méthode réflexive appliquée au problème de Dieu chez Lachelier et Lagneau, 225-226. Es interesante considerar que el camino reflexivo podría ser interpretado como el esfuerzo del hombre como un long detour por las obras divinas, con el que pretende alcanzarlo y por el que Él mismo también deviene.

11 Cf. Grondin, "Paul Ricenur, Méthode réflexive appliquée au problème de Dieu chez Lachelier et Lagneau", 1.

12 Cf. Jean Grondin, “De la dimension métaphysique de l'herméneutique”, en Du sens des choses. L'idée de la métaphysique, 
filosofía sin absoluto a la vista, que movilizaría el manifiesto "rodeo reflexivo" por asuntos que parecen serle extraños -extrañeza operativa en un doble sentido- a Dios y al resto de la obra ricœuriana. ${ }^{13}$ Así en su memoria, coincidirían en el ocultamiento de lo inédito el comienzo temporal y el origen temático desde los cuales Ricœur desarrolló su camino posterior, agnósticamente manifiesto y quizás secretamente metafísico. Esta interpretación parece confirmarse cuando se leen las palabras del propio autor al inicio de su obra. Anticipando el homenaje citado del final, reconoce su interés por la vía metafísica y el problema directriz de Dios, así como por los desafíos que representan para la filosofía religiosa:

He limitado este estudio a Lachelier y a Lagneau; no porque ellos sean las únicas ilustraciones del método reflexivo, sino porque Lachelier y Lagneau son los dos pensadores que han encontrado en el método reflexivo el principio de una metafísica integral. [...] No he dado una misma importancia a todos los aspectos de la obra de estos dos filósofos: como el título lo indica, no contemplo de su pensamiento más que los elementos netamente orientados hacia la investigación religiosa. [...] el problema de Dios ha sido, en el uno y en el otro, la idea directriz de toda meditación; sus reflexiones en apariencia las más alejadas no sirven sino para asegurar los abordajes de una marcha fundamental, que es la búsqueda del absoluto en eso que le es, en apariencia, lo más extraño. ${ }^{14}$

El innegable valor filosófico que la asunción expresa de este punto de partida de juventud ofrece para su camino posterior, vuelve a contrastar con la autointerpretación que Ricœur hace tardíamente de este texto. En su Autobiografía intelectual (1995), significativamente intitulada (si se tiene en cuenta el título de su memoria), Réflexion faite, confirma que esta obra de juventud no encaja con su "madura hermenéutica de sí". Por un lado, la valora positivamente como un primer armisticio en "la guerra intestina entre la fe y la razón” ${ }^{15}$ que acompañó desde el inicio hasta el final todo su itinerario filosófico. Que pensadores tan celosos de la racionalidad y de la autonomía de pensamiento, como Lachelier y Lagneau, le hubieran hecho un lugar privilegiado a Dios en la filosofía, "me satisfizo intelectualmente, [pero aclara enseguida en concordancia con Soi-même comme un autre] sin que ni uno ni otro de estos maestros me invitara a amalgamar la filosofía y la fe bíblica". ${ }^{16}$ Por otro lado, la valora negativamente en tanto estas "incursiones precoces en el camino del Dios de los filósofos prácticamente no tuvieron continuación, a pesar de las imprudentes promesas que pueden leerse en el prefacio de Filosofía de la voluntad." ${ }^{17}$ En su madurez, la

sous la direction de Jean Grondin (Paris:, Presses Universitaires de France, “Chaire Etienne Gilson”, 2013): 143-170. URL: https://www.cairn.info/du-sens-des-choses--9782130625643-page-143.htm; y Jean-Louis Vieillard-Baron, "Les origines spiritualistes de la pensée de Paul Ricœur", Revue des sciences religieuses 92, n. 2 (2018): 245-262.

13 Ricœur afirmará en su madurez que: "No soy un filósofo cristiano [...]. Soy, por un lado, un filósofo a secas, incluso un filósofo sin absoluto. Y, por otro lado, un cristiano de expresión filosófica, como Rembrandt es un simple pintor y un cristiano de expresión pictórica y Bach un simple músico y cristiano de expresión musical." Paul Ricœur, Vivant jusquà la mort, suivi de Fragments (Paris: Seuil, 2007), 107.

14 Ricœur, Méthode réflexive appliquée au problème de Dieu chez Lachelier et Lagneau, 29-30. Esta consideración se mantiene, a lo largo de toda la memoria, donde Ricœur insiste en la necesidad de preguntarse sobre qué metafísica se apoya cada autor (64) y cómo establecer los fundamentos metafísicos sobre los que se sustenta la fe moral en cada caso (85).

15 Ricœur, Autobiografía intelectual, 13.

16 Ricœur, Autobiografía intelectual, 17.

17 Ricœur, Autobiografía intelectual, 17. Tales promesas imprudentes refieren a los tomos II y III de Philosophie de la volonté. Ante la aparición del problema ético de la falta que se da ante Dios y, por tanto, la correlativa afirmación de la Trascendencia, dice Ricœur: "Pero, en ese momento, la falta, única capaz de poner sobre su verdadero terreno 
profesión de agnosticismo filosófico y la apreciación de precocidad e imprudencia respecto al camino iniciado en su juventud, dan la idea del reconocimiento de errores cometidos por un filósofo debutante y de una vía juiciosamente interrumpida. La cuestión metafísica de Dios no sería para el Ricœur maduro ni el único problema filosófico, ni la idea directriz íntimamente vinculada al desarrollo de una metafísica integral y al método de la filosofía reflexiva que continuará practicando constantemente. La decisión de tal abandono o toma de distancia -no paulatina, sino abrupta- en el acto de su pensamiento parece haber sido una decisión, al mismo tiempo, profundamente personal y filosófica, que podría pensarse como el primer giro de su filosofía, el primer corte exigido para cualquier injerto futuro. Creo que se enfrenta entonces uno de los escritos más conflictos de Ricœur para sí mismo, a tal punto que lo ha dejado por fuera del rodeo reflexivo -en una vía que tal vez ha pretendido no ser lo suficientemente larga- para dar un sello a la totalidad de su obra filosófica.

La problemática que marcha respecto a esta primera cuestión establece como objetivo de este trabajo: reconstituir los inicios de esta paradoja bajo la pregunta ¿qué ideas de metafísica y, correlativamente, de Dios tiene Ricœur en su memoria? La presentación de estas ideas iniciales pretenderá esclarecer la génesis paradójica de su camino filosófico maduro a través de tres puntos de análisis de su memoria: 1) El credo filosófico con el que inicia; 2) Las decisiones que toma a lo largo de ella sobre las ideas mencionadas; y 3) La estructura de una conclusión imposible con la que finaliza.

\section{El credo filosófico de Ricœur}

Ricœur inicia con su memoria el largo camino filosófico que se objetivará en la totalidad de su extensa obra. La paradoja, allí anudada, se anuncia en la primera línea del prólogo. Se trata "ante todo de un esfuerzo de reconstitución histórica”. ${ }^{18}$ Reconstruir el pensamiento de Lachelier y Lagneau a través de la

al problema ético, es asimismo la única capaz de deponer la ética considerada como el orden cerrado de la ley. La falta se encuentra en relación con Dios, está ante Dios, y la subjetividad es superada por su propio exceso. [...] La abstracción de la Trascendencia promueve tantas dificultades como la abstracción de la falta. En efecto, ambas abstracciones son inseparables. La experiencia integral de la falta y su contrapartida mítica, la imaginación de la inocencia, son estrechamente solidarias de una afirmación de Trascendencia: por una parte, la experiencia integral de la falta es la falta experimentada como un estar ante Dios, es decir, el pecado. Por ello, no es posible disociar falta y Trascendencia. Pero, sobre todo, la Trascendencia es aquello que libera la libertad de la falta. Así viven los hombres la Trascendencia: como purificación y redención de su libertad, como salvación. La Trascendencia resplandece para nosotros en relación a un mundo espiritual que tiene lesiones reales. Todos los otros accesos, que pueden parecer un camino más corto, son en realidad extraños a la prueba concreta de la Trascendencia, que significa nuestra integridad recobrada. La cautividad y la redención de la libertad son el único y el mismo drama." Paul Ricœur, Philosophie de la volonté I (Paris: Aubier, 1950), 20-21 y 25-26.

18 Ricœur, Méthode réflexive appliquée au problème de Dieu chez Lachelier et Lagneau, 29. El término “esfuerzo" es recurrente (aparece 31 veces) y tomado de Maine de Biran se mantiene hasta sus obras tardías. En la idea de metafísica que hay en la memoria, el esfuerzo refiere, sobre todo, a un intento filosófico humano no consumado a causa de una resistencia divina que induce al necesario inacabamiento. Respecto a la idea de inacabamiento, como idea directriz de su filosofía, en La memoria, la historia y el olvido (2000), obra que algunos consideran su testamento filosófico, Ricœur le escribió a Catherine Goldenstein -la asistente que lo acompañaría hasta el final de sus días-: "Al termino del trayecto que hice durante décadas desde la vida hacia los textos y el discurso, usted me ayuda, querida Catherine, a hacer el trayecto inverso: de los libros hacia la vida, y creo que este libro que terminaré este invierno llevará la huella de este movimiento de retorno." Le transmite que la última palabra del libro será "inacabamiento" en una suerte de poema que dice así: "Bajo la historia, la memoria y el olvido,/ Bajo la memoria y el olvido, la vida/Pero escribir la 
idea directriz del problema de Dios. El término esfuerzo ya indica el carácter de un intento filosófico que, según Vallée, tiene la importancia de ser la primera explicación por parte de Ricœur del idealismo de Kant y Hegel a través de la recepción de Lachelier y Lagneau. ${ }^{19}$ La razón de centrarse en estos últimos -lo recuerdo- es que han encontrado en el método reflexivo el principio de una "metafísica integral". Ricœur utiliza esta segunda expresión para indicar una concepción integral del ser que no sólo sea una toma de conciencia filosófica de la búsqueda del fundamento absoluto que es la cuestión de Dios, sino que, a partir de los fracasos que provoca y el inacabamiento a la que obliga en el esfuerzo del pensamiento por alcanzarla, entienda al ser como una voluntad y libertad, productoras de lo real y de sí mismo. ${ }^{20}$ Se anuncia así una metafísica que ya integra en sí los problemas del querer y de la moral que no sólo la limitan, sino que la obligarán a dar el paso a una doble trascendencia: inmanente y Trascendente. Y “¿cómo se encuentra esa física y metafísica integral?" ${ }^{21}$ No a través del método deductivo que no es un método de investigación, sino a través del análisis (que remite al método Metafísico de Spinoza -palabra que Ricœur escribe con mayúscula-), que partiendo de hechos particulares, los descompone y descubre en ellos lo que les falta y suponen. El método reflexivo y sus métodos vecinos como el método de inmanencia, ponen en práctica este análisis. Lachelier y Lagneau lo aplican al problema de Dios, que es precisamente directriz, porque en su búsqueda muestra los límites de esta metodología regresiva y de su doctrina inmanente, obligando a la integralidad que, en última instancia, según Ricœur, es la necesaria complementariedad de una metodología prospectiva y de una doctrina trascendentes. Al final del prólogo, la descripción y adhesión a este método reflexivo concluye con un pequeño "credo filosófico", donde Ricœur formula un principio paradójico que le parece la "verdadera perspectiva de la investigación filosófica” y -creo- la condición primera de esa metafísica integral: la inmanencia de la reflexividad conlleva siempre cierta trascendencia.

Si la historia de la filosofía puede a veces erigirse en disciplina autónoma, y devenir una erudición libre, normalmente es la ocasión de una profundización personal. Si es imposible recibir el pensamiento de los demás sin que él encuentre en la conciencia direcciones latentes y suscite reacciones específicamente personales, en compensación, no es cierto que uno pueda descubrirse a sí mismo sin descubrir a los demás. No se desciende a sí más que saliendo de sí. No se encuentra más que perdiéndose. No se accede a lo Mismo más que por el Otro. Toda inmanencia envuelve alguna trascendencia. ${ }^{22}$

vida es otra historia./Inacabamiento" Paul Ricœur, La memoire, l'histoire, l'oubli (Paris: Le seuil, 2000), 657. La citada carta termina: "Le agradezco por ayudarme a inacabar mi vida." Ricœur, Carta a Catherine Goldenstein, agosto de 1998, citada por F. Dosse, Paul Ricœur. Los sentidos de una vida (1913-2005) (Buenos Aires: FCE, edición revisada y aumentada, 2008), 736.

19 Cf. Marc-Antoine Vallée, "Le premier écrit philosophique de Paul Ricœur". Méthode réflexive appliquée au problème de Dieu chez Lachelier et Lagneau, Études Ricœur iennes / Ricœur Studies 3, n. 1 (2012): 149.

20 La expresión "metafísica integral” refiere, como señala Greisch, a una fórmula célebre del artículo de Lachelier, "Psychologie et Métaphysique": "Lêtre tel que nous le concevons n’est pas d’abord une nécessité aveugle, puis une volonté, qui serait enchaînée d'avance par la nécessité, enfin une liberté, qui n’aurait plus qu’à constater l'existence de l'une ou de l'autre. Il est tout entier liberté, en tant qu'il se produit lui-même, tout entier volonté, en tant qu'il se produit comme quelque chose de concret et de réel." Jules Lachelier, Du fondement de l'induction suivi de Psychologie et Métaphysique et de Notes sur le pari de Pascal (Paris: Félix Alcan, 1924), 170; citado por Greisch en Ricœur, Méthode réflexive appliquée au problème de Dieu chez Lachelier et Lagneau, 15.

21 Ricœur, Méthode réflexive appliquée au problème de Dieu,149.

22 Ricœur, Méthode réflexive appliquée au problème de Dieu, 22-23. 
A la luz de este principio, donde resuena toda su filosofía futura, Ricœur se dedica a reconstituir el idealismo filosófico de Lachelier y Lagneau en relación con el método reflexivo adoptado. ${ }^{23}$ Su punto de partida es el idealismo metodológico de ambos autores. Para Ricœur, una "filosofía integral" es una filosofía del pensamiento que requiere del método reflexivo como "redoblamiento del pensamiento sobre él mismo en vistas de saber su propia naturaleza"24; redoblamiento que abre a la vida moral a partir del esfuerzo siempre incompleto por alcanzar a Dios en la inmanencia. En este sentido, adhiere a la crítica que Lagneau dirige contra la prueba moral de la existencia de Dios de la metafísica kantiana (fundada sobre el hecho de la existencia de la ley moral en mí) por no ser una prueba ni lo suficientemente estricta y directa -al no analizar sus condiciones-, ni ser una prueba religiosa, es decir, a la vez moral y metafísica que resulta, en verdad, de la reflexión del pensamiento sobre él mismo, en vistas de comprenderse, de comprender las necesidades a las que obedece cuando intenta demostrar la existencia de Dios y los obstáculos que no puede superar en esta demostración. ${ }^{25}$ Ricœur comulga con ese espíritu de fondo del método reflexivo del idealismo, pues considera que es el único método que permite conocernos a nosotros mismos y responder a la cuestión fundamental de la filosofía “¿qué soy yo (je), yo (moi) que pienso el mundo?" ${ }^{26}$ Pero a causa del esfuerzo recurrente por pasar de un idealismo metodológico al idealismo doctrinal y, sobre todo, la falta de reconocimiento de límites frente a la cuestión filosófica de Dios, Ricœur expresa, en cercanía a Lagneau, con qué idea de metafísica no está de acuerdo:

Si el ideal de la metafísica es comprender la unidad del Ser, felicitémonos por el fracaso de la metafísica, porque este fracaso marca el lugar de la moral. Es porque nuestra naturaleza siempre permanece por debajo de Dios; es porque hay en el Ser algo que no debería ser, que el esfuerzo tiene un sentido, que el sacrificio nos llama y que una alternativa nos urge. El esfuerzo moral, la acción que remonta sin cesar la pendiente de la mala voluntad, vive en la distancia que la metafísica no puede cerrar entre Dios y la naturaleza: es porque hay no-Ser que nuestra vida debe ser un renunciamiento y un sacrificio, es porque Dios no es, que es necesario, por nuestro esfuerzo, que Dios devenga (devienne). La vida moral es una Alternativa en la medida en que el Monismo de la metafísica es inacabado. ${ }^{27}$

23 El método reflexivo con este primer ejercicio del método de confrontación crítica, Ricœur lo practicará hasta sus obras de madurez. No hay sólo que reconstituir históricamente y comparar ambos filósofos, sino mostrar las fisuras de su pensamiento donde se insertará la crítica. Según Vallée en este método se encuentra la originalidad y "el verdadero porte filosófico de la obra para los estudios ricœurianos actuales." Vallée, "Le premier écrit philosophique de Paul Ricœur. Méthode réflexive appliquée au problème de Dieu chez Lachelier et Lagneau", 148-149.

24 Ricœur, Méthode réflexive appliquée au problème de Dieu, 35.

25 Lagneau critica la prueba de Kant afirmando: "cette preuve de Kant nétait pas suffisamment directe, qu’elle reposait sur des postulats, c'est-à-dire sur des exigences de la raison et sur des besoins de la nature, et que, par conséquent, elle les présupposait; que Dieu nétait pas atteint directement par la réflexion sur l’acte même de la pensée, sur les conditions de la pensée; que le lien qui rattache Dieu à la réalité et à la pensée nest pas encore tellement étroit dans cette preuve que l'on ne puisse encore concevoir l'une et l'autre sans lui." J. Lagneau, De la existence de Dieu (Paris: Félix Alcan, 1925), 43-44. Lachelier y Lagneau resuelven el problema de existencia de Dios mostrando que no es un verdadero problema. En el caso de Lachelier su realidad está perfectamente asegurada en tanto no es un objeto de suposición, ni una cosa extraña al espíritu que deba demostrarse, sino el poder mismo del pensamiento que se descubre en nuestra inmanencia. Según Lagneau, la existencia de Dios no sería un objeto de demostración teórica, ya que sólo importa hacer el esfuerzo de explicitación o develamiento que compromete una transformación de nuestra inteligencia habitual sobre lo que es Dios. No se accede a él concibiéndolo como un ente supremo, sino al pensarlo como un acto, una pura libertad, el acto mismo de posición en el fundamento de todo pensamiento.

26 Ricœur, Méthode réflexive appliquée au problème de Dieu, 158.

27 Ricœur, Méthode réflexive appliquée au problème de Dieu, 223-224. 
A la luz de esta crítica a una metafísica completa sin fracaso y sin finitud, es que Ricœur adopta tres tomas de posición -tal como señala Greisch en el prólogo de la memoria- respecto al modo de practicar el método reflexivo. Creo estas tres decisiones bosquejan su comprensión de la metafísica integral y de la cuestión correlativa de Dios, así como ciertas implicancias religiosas que ellas conllevan.

\section{Las decisiones por las preguntas}

La primera decisión es doble: de adhesión y oposición crítica a las posturas de Lachelier y Lagneau. 1) Al rechazar toda aproximación deductiva a priori de una filosofía del espíritu, Ricœur adhiere -como se ha visto- a la caracterización del método como un análisis. El método reflexivo de Lachelier y Lagneau no parte de una idea primaria de la cual se deduzca toda la estructura del espíritu sino de experiencias concretas del pensamiento que se analizan para remontarse a su condición fundamental de posibilidad. Es decir, no parten de una idea de Dios sino que llegan a ella. Un punto de llegada que nunca se alcanza tiene un punto de partida que siempre es donación. Al remontarse por la vía reflexiva e inmanente a la búsqueda del absoluto, Lachelier y Lagneau lo hacen por donaciones que en apariencia le son extrañas a esta cuestión. En el caso de Lachelier, es el acto de inducción y en el caso de Lagneau, la experiencia de percepción; formas particulares del pensamiento a partir de las cuales se remontan al Todo del pensar. Su método reflexivo es por eso "una marcha hacia atrás, un estudio regresivo" 28 que no crea nada, sino que es una toma de conciencia, un pasaje de lo implícito a lo explícito, donde Dios se descubre en el seno mismo del pensamiento, en la inmanencia más radical, siendo su fundamento. Dice Ricœur, se trata de una "búsqueda de la condición a partir de lo condicionado"29 que tarde o temprano llega al absoluto, incluso si -y de allí su propio talón de Aquiles-, cuando se lanza en busca de Dios, el método reflexivo se niega a recibir una luz extraña a nuestro pensamiento. Por eso si bien Ricœur admite con ambos la necesidad de superar una estricta ortodoxia kantiana sobre las condiciones a priori de la experiencia, las formas de conocimiento verdadero y la prueba moral de la existencia de Dios, se resiste también a la idea de una metafísica que ofrece la solución especulativa del idealismo absoluto: "Si la razón es el absoluto, ella debe no solamente bastarse a sí misma, sino explicar todas las cosas." Esta exigencia de absoluto que el idealismo doctrinal no llega a satisfacer jamás, hace fracasar incluso a una metafísica que pretende ser integral pero donde la cuestión de Dios no conduce a la trascendencia ni da paso a la moral. En este matiz de su adhesión, este Ricœur, aun pre-fenomenológico, establece los cimientos de una metafísica necesariamente genética, pero verdaderamente integral, pues en su marcha regresiva deberá convertirse en acogida o recepción de esa trascendencia que conlleva toda inmanencia. 2) Ricœur se opone a la caracterización intelectualista del método reflexivo como método psicológico de introspección del pensamiento que no se interesa por su dimensión subjetiva y singular, sino por el pensar bajo la forma impersonal y universal, esforzándose por explicar su naturaleza y estructura que hacen posible el conocimiento objetivo. ${ }^{31}$ La práctica del método reflexivo conlleva la oposición inicial de sensibilidad y razón, de individuo e impersonalidad, en tanto no es una toma de conciencia del pensamiento individual por parte de una persona, sino un análisis impersonal de la intelectualidad

28 Ricœur, Méthode réflexive appliquée au problème de Dieu, 47.

29 Ricœur, Méthode réflexive appliquée au problème de Dieu, 15.

30 Ricœur, Méthode réflexive appliquée au problème de Dieu, 145.

31 Al respecto Cf. Jean-Louis Vieillard-Baron, “Les origines spiritualistes de la pensée de Paul Ricœur", 245-262. 
universal. Por eso mismo, el idealismo doctrinal tiende al intelectualismo y al impersonalismo, y considera que el hombre no es más que espíritu. La crítica de Ricœur es que: "Los partidarios del método reflexivo olvidan a menudo que no soy solamente un individuo, sino una persona [...] no soy una novela mediocre de apariciones y acontecimientos; soy un drama y un destino. Eso es mi persona que tiene la responsabilidad del pensamiento, la carga de la duda y la iniciativa de la buena voluntad". ${ }^{32}$ Creo que esta decisión de oposición de Ricœur completa los cimientos de esa metafísica integral que, a causa de su inacabamiento cuando se encuentra con la trascendencia inmanente y Trascendente, debe abrirse a la vida moral y por eso sólo puede concretarse como un acto personal.

La segunda toma de posición parte de ese intelectualismo del idealismo reflexivo. Si el pensamiento no tiene nada que ver con la sensación ni con la individualidad, es también distinto de lo social y colectivo. Coincidiendo aquí con Lachelier (y Fichte), contra Durkheim y Brunschvicg, Ricœur sostiene que el fenómeno religioso no debe aprenderse originariamente como un hecho social total, sino como un esfuerzo interior del alma -nuevamente un camino genético- que buscando desprenderse de todo lo que ella no es, en expresión de su libertad, llega a rodear la esencia de la religión, pues "la razón sola, no la sociedad, es la guardiana de la verdadera trascendencia." ${ }^{33}$ Desde esta posición, parece quedar en correspondencia el carácter personal del esfuerzo religioso y el del acto reflexivo de una metafísica integral, desde la cual Ricœur evalúa las fisuras del intelectualismo de Lachelier y Lagneau, y las implicancias de sus propios esfuerzos religiosos. El primero es más cerrado y alcanza a un Dios racional (por lo tanto, no habría integralidad). El segundo hace un esfuerzo de crítica a la evidencia intelectual y tiende a sobrepasarla en el amor y la acción que son "una encarnación más alta de la razón”. ${ }^{34}$

Finalmente, Ricœur asume una tercera y última decisión que se corresponde con la toma de posición religiosa descripta y refigura su primera adhesión al método reflexivo, dejando entrelazadas sus tres decisiones: "el pensamiento es principio teórico del ser; el pensamiento es ideal práctico de vida. La filosofía reflexiva es el nudo mismo de estos dos puntos de vista. Practicar el método reflexivo no es un juego de ideas, sino una disciplina de vida." ${ }^{35}$ Tal método invita a una conversión en tanto pretende ser un método para arribar a la vida bienaventurada: "Él es una purificación, una ascesis, una conversión."36 ¿A qué nos convierte? ¿en qué nos purifica? Ricœur lo explica con Lachelier, mostrando que el idealismo debe proyectarse en la esperanza de la fe religiosa ("nuestra vida interior se prolonga más allá de la filosofía y se expande en la fe: eso es solamente la vida religiosa" ${ }^{37}$ ) y con Lagneau en tanto el acto más elevado del pensamiento consiste en enfrentarse a lo incomprensible y por eso prolongarse sobre el plano

32 Vieillard-Baron, "Les origines spiritualistes de la pensée de Paul Ricœur”, 39. Greisch afirma que "Drame et destinée: les deux vocables ont du poids et expliquent l'ascendant de Gabriel Marcel sur le jeune Ricœur." Ricœur, Méthode réflexive appliquée au problème de Dieu,18.

33 Ricœur, Méthode réflexive appliquée au problème de Dieu,19. Ricœur coincide con Lachelier cuando afirma: "La religion consiste pour lâme qui en est capable dans un effort individuel et solitaire pour s'affranchir et se déprendre de tout ce qui n'est pas elle et de tout ce qui, en elle, n'est pas sa liberté même [...] La religion ignore le groupe ; elle est un effort intérieur et, par suite, solitaire."J. Lachelier, "Sur le problème religieux et la dualité de la nature humaine", Société française de philosophie, séance du 4 févr. (1913, Euvres t. II): 167-171.

34 Ricœur, Méthode réflexive appliquée au problème de Dieu, 44.

35 Ricœur, Méthode réflexive appliquée au problème de Dieu, 50.

36 Ricœur, Méthode réflexive appliquée au problème de Dieu, 51.

37 Ricœur, Méthode réflexive appliquée au problème de Dieu, 52. 
de la acción buena y del amor. El método reflexivo debe asumir el riesgo de la prolongación prospectiva de la cuestión metafísica de Dios por caminos extraños, indicados en las preguntas que cierran la introducción de la memoria:

“Es suficiente instalarse pura y simplemente en el universo de la ciencia y la justicia? ¿La reflexión intelectual tiene su fin en sí misma? ¿O nos enfrenta cara a cara con un enigma siempre recurrente, que nos invita a correr el riesgo de la fe religiosa en un Dios revelado, o a trascender la comprensión por anticipaciones más oscuras, condenadas por cierto a no ser jamás saturantes y exhaustivas?"38

\section{La estructura de una conclusión imposible}

A partir de la reflexión crítica de este análisis histórico, se ensayará un análisis proyectivo de la idea de metafísica y de Dios en este muy joven Ricœur. Para ello hay que reconocer que las filosofías de Lachelier y Lagneau tienen el problema de realizar "un esfuerzo por hacer coincidir un método de inmanencia con una filosofía de la inmanencia". ${ }^{39}$ Ricœur no critica la filosofía de la inmanencia más que en su intento de acabamiento, pues "no creo que un método de inmanencia pueda ser suficiente por sí mismo. Una razón más fuerte aún, una filosofía de inmanencia, para no ser falsa, es radicalmente incompleta." ${ }^{40} \mathrm{La}$ asunción del método reflexivo conduce entonces a reconocer no la necesidad del agnosticismo, sino de la inconclusión o inacabamiento de la metafísica y la necesidad de prolongar su camino por rodeos que parecen serle extraños como el de la moral. Por eso la conclusión de la memoria se divide en dos partes cuya estructura interna se corresponde -creo-, mostrando las líneas proyectivas de una metafísica y de la cuestión de Dios en ella. Por un lado, examina el método de inmanencia, (punto A) y, por otro, la doctrina de la inmanencia (punto B). Ambas guardan una proporción de igualdad, mostrando al inicio de cada una la "necesidad de un método de inmanencia" (punto A.1), y la "necesidad de una doctrina de la trascendencia” (punto B.1). Expongo las proyecciones metafísicas que conlleva cada una.

A) El método de inmanencia se prolonga: 1) en su necesidad como método reflexivo; 2) en la necesaria relación a la que obliga entre reflexión y acción, y 3) en su comprensión última como recepción. 1) A pesar de las afirmaciones formuladas al inicio de la obra, Ricœur reconoce que la trascendencia no es evidente, pues incluso la trascendencia inmanente, es decir, la cosa en sí trascendente a nuestro espíritu está siempre fuera del alcance humano: "Ya sea que exista una trascendencia o no, es imposible establecerse allí de antemano." ${ }^{41}$ Lo que sería evidente entonces sería nuestra finitud, por eso "yo creo que la reflexión consiste mucho más en descubrir en nosotros estas ausencias que estas presencias." ${ }^{42} \mathrm{La}$ necesidad del método reflexivo radica en mostrar esa negatividad que perfila: a) la idea de trascendencia

38 Ricœur, Méthode réflexive appliquée au problème de Dieu, 52-53.

39 Ricœur, Méthode réflexive appliquée au problème de Dieu, 225.

40 Ricœur, Méthode réflexive appliquée au problème de Dieu.

41 Ricœur, Méthode réflexive appliquée au problème de Dieu, 226.

42 Ricour, Méthode réflexive appliquée au problème de Dieu, 226. Vallée reconoce que la primera continuidad evidente con la filosofía madura de Ricœur es que en su primer escrito frente a los límites del idealismo de Lachelier y Lagneau el joven Ricœur ya se orienta hacia una filosofía de la finitud que encontrará una prolongación natural en el pensamiento hermenéutico. Cf. M-A. Vallée, "Le premier écrit philosophique de Paul Ricœur. Méthode réflexive appliquée au problème de Dieu chez Lachelier et Lagneau”, 152. 
como ausencia, incluso Ausencia de Dios y de su no existencia, que podría ser prueba moral, en el sentido de ponernos a prueba en la humildad de nuestra libertad y frente al mal que ella causa, y b) la necesidad de una metafísica de esa ausencia o falta siempre supuesta en la práctica de la reflexión teórica, en tanto la búsqueda de Dios engloba nuestra vida entera. En este sentido, se comprende mejor por qué Ricœur afirma que la satisfacción y exhaustividad intelectual, es decir, la saturación del entendimiento por parte de ideas claras y distintas, es "la raíz del verdadero ateísmo." ${ }^{33}$ 2) A partir de los límites descubiertos por el método reflexivo, se reafirma la importancia del carácter integral de una metafísica. Lagneau es el que nos resguarda, según Ricœur, de la ilusión de que la reflexión sea una búsqueda puramente especulativa de la verdad, y muestra que también es una acción. La reflexión en acción sería la vocación práctica de todo esfuerzo metafísico. Al igual que las ausencias que descubre la reflexión, frente a la acción cabe reconocer su modesta escala: "no tenemos en nosotros para crear el Ser sino de alguna manera para recibirlo. [...] No tenemos solamente que hacer nuestra vida, sino en cierta medida recibirla."44 3) La acción modesta y humilde media la reflexión con la recepción, en tanto tenemos que "abrirnos a aportes exteriores, dilatar nuestra realidad humana, al contacto con otras realidades parciales. [...] No tenemos solamente que profundizarnos, sino abrirnos. ${ }^{35}$ El pensamiento se apoya sobre algo que no viene de él mismo, y que debe acoger e interiorizar, para recrear. Así ensaya una escala metafísica de tres grados de recepción del ser donde dos de ellos se caracterizan como amor: 3.1) Para ser nosotros mismos tenemos necesidad de la primera recepción más humilde que es la de las cosas materiales, cosas que no creamos y son una verdadera alteridad para el pensamiento. El método reflexivo nos conduce a través del camino de Agustín que tanto agradaba a Blondel, por una metafísica de la recepción de la trascendencia en la materia: encontrarnos, encontrando al mundo, perdernos en las cosas del mundo para poder rencontrarnos. ${ }^{46}$ 3.2) La recepción no se acaba en las cosas sino se prolonga al mundo de las personas, y "el Amor es esta búsqueda y esta acogida superiores". ${ }^{47} \mathrm{El}$ amor necesita la base esencial de la noción de persona porque "amar es salir de sí, esto es descubrir una alteridad, interiorizar a otro exteriorizándose a sí-mismo". ${ }^{48} \mathrm{La}$ metafísica de la recepción de la trascendencia personal exige la acción del amor. 3.3) La recepción última es el descubrimiento del absoluto que no es sólo un desenvolvimiento de nuestras pre-posesiones, ni un simple pasaje de lo implícito a lo explícito, sino también una dilatación de nuestro ser por la intrusión de alguna otredad: "Tal vez hay una acogida suprema, un amor supremo, que nos saca de nosotros mismos, y para algunos, de todas sus riquezas anteriores. Quizás haya un despojo último que finalmente nos haga coincidir con nuestra riqueza anterior. La oración del hombre, la gracia de Dios, esta es la reunión final que el hombre tal vez debería hacer para completar su sabiduría y lograr su filosofía." ${ }^{49}$ El encadenamiento de los adverbios y verbos condicionales (tal vez, quizás, debería) indican que la metafísica integral "se

43 Ricœur, Méthode réflexive appliquée au problème de Dieu, 227.

44 Ricœur, Méthode réflexive appliquée au problème de Dieu, 230.

45 Ricœur, Méthode réflexive appliquée au problème de Dieu, 231.

46 La frase agustiniana citada por Ricœur es: “Ab exterioribus ad interiora, ab interioribus ad superiora”. Ricœur, Méthode réflexive appliquée au problème de Dieu, 232.

47 Ricœur, Méthode réflexive appliquée au problème de Dieu, 232.

48 Ricœur, Méthode réflexive appliquée au problème de Dieu, 232. Allí dice que no descubrimos nuestra identidad más que por el camino de nuestra diferencia.

49 Ricœur, Méthode réflexive appliquée au problème de Dieu, 233. A pesar de la cercanía e influencia reconocida respecto a la metodología analítica, es difícil determinar hasta qué punto el joven Ricœur recibió inspiración aquí de la teoría de los tres amores que Spinoza desarrolla en su Tratado Breve, en relación con los modos de conocimiento que serían en Ricœur los tres modos de recepción antes descriptos. 
completaría y lograría" en la doble recepción religiosa de la Trascendencia de Dios: la ascendente de la plegaria que el filósofo debería rezar y la descendente de la gracia divina que debería acoger.

B) La doctrina de inmanencia se prolonga: 1) en la necesidad de una doctrina de la trascendencia; 2) en la necesaria relación a la que obliga entre trascendencia y personalismo, y 3) en su comprensión última de los grados del Ser y del no-Ser. 1) La necesidad del método de la inmanencia pone de manifiesto por su inacabamiento la necesidad de una doctrina de la trascendencia. En tanto la inmanencia, para ser verdad, "permanece incompleta, hay una doctrina de la trascendencia dentro de una doctrina de inmanencia." ${ }^{50}$ Eso implica que "un método reflexivo debe apoyarse en un método prospectivo, que un método de inmanencia debe alimentarse de la recepción de alguna trascendencia." ${ }^{51}$ Para Lachelier y Lagneau tal cosa no es posible, pues su idealismo es completo, es decir, su metafísica pretende la unidad del ser, de Dios y naturaleza, y Ricœur -lo recuerdo- celebra su fracaso. Por el contrario, propone que, sin negar la unidad del ser (ahora se verá en qué sentido), haya una metafísica de grados del ser entre la materia y el espíritu, en el que se supone que el último grado sería la Trascendencia inmanente; por eso se abre la pregunta ¿quién es Dios en esta metafísica? Confrontando con la propia fórmula que había utilizado anteriormente, "es porque Dios no es, que es necesario, por nuestro esfuerzo, que Dios devenga (devienne)", ahora concluye sobre Dios: "Por nuestra reflexión, Dios deviene. Pero en realidad Dios no necesita profundizarse, porque Dios es. Sin duda, esta existencia de Dios, distinta de este devenir y de este esfuerzo, no es algo externo a nuestra conciencia; existe en nosotros, en el estado implícito, como una especie de Inconsciente, y eso es suficiente para que la trascendencia no contradiga la inmanencia." ${ }^{2}$ Dios es un pensamiento que, en tanto no es conocido, es trascendente. Es nuestro ser en la medida en que este ser no es Consciencia. "Así, la mera idea de una transición de lo implícito a lo explícito envuelve la idea de trascendencia." ${ }^{53}$ Nuestro esfuerzo reflexivo por el que buscamos alcanzar a Dios, nos descubre la distancia de lo que hacemos devenir, esa plenitud del ser que "es una auténtica trascendencia." ${ }^{54}$ Sobre ese Absoluto tan distante que es $y$, a la vez, tan inmanente a nuestro esfuerzo de hacerlo devenir porque no es, cuya recepción exige prolongar y completar ese esfuerzo en el doble camino de recepción religiosa, Ricœur afirmará: "Dios es dado en una oscura necesidad, la gracia que yace latente en la naturaleza, el amor que duerme en las profundidades del deseo, la nostalgia de Dios. Si uno avanza más adelante en esta dirección, que de hecho está muy cerca del punto de vista de M. Blondel, debe decir que la verdadera guía de nuestro devenir, de nuestro esfuerzo intelectual y moral, está en esta pre-posesión, esta anticipación, este saber implícito que duerme en las regiones más oscuras del hogar cristalizado de nuestras ideas claras." ${ }^{55}$ Ese Dios proto-fuente que Ricœur describe con tantas metáforas, adverbios y tiempos condicionales, es el que da unidad al método reflexivo como principio de la metafísica y la moral, y las cumple en el inacabamiento de la religión: "Quizás, por el contrario, que este oscuro conocimiento, que duerme en esta problemática franja de nuestra alma, en este halo que aún no ha sido absorbido por la reflexión, y que es vida, acción, contiene normas, e incluso una verdadera revelación natural, una

50 Ricœur, Méthode réflexive appliquée au problème de Dieu, 233.

51 Ricœur, Méthode réflexive appliquée au problème de Dieu, 234.

52 Ricœur, Méthode réflexive appliquée au problème de Dieu, 237.

53 Ricœur, Méthode réflexive appliquée au problème de Dieu, 237.

54 Ricœur, Méthode réflexive appliquée au problème de Dieu, 237-238.

55 Ricœur, Méthode réflexive appliquée au problème de Dieu, 239. 
voluntad profunda que significa algo decisivo sobre nuestro destino." ${ }^{6}$ Sobre esta idea de Dios se perfila la metafísica integral que debe asumir su Ausencia y su Nostalgia alcanzadas por la reflexión y la exigencia moral correspondiente que se impone a la acción como esfuerzo. Adhiriéndose a otra cita de Blondel, Ricœur dice a este respecto: "cada esfuerzo que se intenta para satisfacer exigencias anteriores que se manifiestan espontáneamente al pensamiento, revela las exigencias posteriores que se imponen moralmente a la acción." ${ }^{57}$ Una reflexión metafísica que exige al mismo tiempo la moral en la acción, tiene por objeto una Trascendencia que, más tarde, siguiendo a Jaspers, fascinará a Ricœur y será la ocasión para elaborar una filosofía de la trascendencia que sin llegar a ser una filosofía de la religión ni una filosofía teológica, pretenderá ser una poética integral del ser como poética de la voluntad. 2) La trascendencia de Dios está íntimamente unida al personalismo y a la inmanencia porque ella "no aparece plenamente al hombre más que en la perspectiva de una filosofía de la Persona." ${ }^{58}$ Ricœur explica la trascendencia como una intensificación de nuestra originalidad personal y de la irreductible distancia que permanece entre el pensamiento humano y divino: "la persona es el interés supremo del mundo, y Dios es el principio de la Persona tanto como de la unidad. La síntesis de impersonalidad y personalidad, de verdad y amor, de la norma y el Fiat creativo, es para nosotros incomprensible." ${ }^{59}$ ¿Cómo podría ser entonces una metafísica inconclusa de la unidad sin síntesis? 3) La proyección final de este punto B queda formulada expresamente en clave de esta pretendida metafísica. En correspondencia con la escala de los tres grados de recepción del ser que implicaba el amor (proyección última del punto A), Ricœur examina los grados del Ser y la posibilidad de que haya grados del no-Ser, es decir, de mal. De este modo, la formulación entrecruza la clave ética y metafísica: la recepción del ser que da lugar a la acción del amor y el problema del mal que moviliza la reflexión como recepción. Para examinar esto, la metafísica requiere de una reubicación antropológica: "Esta trascendencia de Dios a la persona humana tiene sentido cuando reubicamos al hombre en el universo." ${ }^{60}$ Se trata de la necesidad de la diversidad y de la unidad humana y de seres para concretar, no una síntesis, sino lo que Ricœur llama una comunión:

El amor no llega al ser íntimo más que a través de los demás. La unidad y la diversidad de los espíritus también son esenciales para el Amor: una fusión total de las personas crearía una conciencia colectiva y no esa comunión que crea diversidad y unidad en proporciones iguales. Esta comunión es la culminación de una tendencia latente en todo el universo. Esta diversidad de espíritus existe para todos los grados del mundo. [...] Hay una verdadera diversidad hasta en la materia. ${ }^{61}$

56 Ricœur, Méthode réflexive appliquée au problème de Dieu, 239.

57 Maurice Blondel, "Le point de départ de la recherche philosophique", Annales de philosophie chrétienne, juin (1906): 234-235.

58 Ricœur, Méthode réflexive appliquée au problème de Dieu, 240. Así señalando la inmanencia impersonal que subyace a la trascendencia personal, afirma que "La trascendencia, que no niega la verdadera interioridad de los pensamientos, tiene como base real el personalismo.” Ibídem, 241-242.

59 Ricœur, Méthode réflexive appliquée au problème de Dieu, 242.

60 Ricœur, Méthode réflexive appliquée au problème de Dieu, 242. Si bien Ricœur no utiliza jamás en su memoria el término "filosofía primera", en vistas del lugar que le otorgará en algunas ocasiones a su antropología, me pregunto si no estamos aquí ante la circunstancia metafísica originaria y fundacional por la cual su filosofía llega a configurarse como una antropológica fundamental.

61 Ricœur, Méthode réflexive appliquée au problème de Dieu, 242-243. 
Una metafísica de los grados del ser implicaría entonces el intento filosófico de una metafísica de la diversidad y de la recepción, no de la síntesis del ser que no nos está permitida; metafísica que se cumpliría gracias a la reubicación antropológica ofrecida por el método reflexivo, tal como lo explica Ricœur:

Al interiorizar el universo a través del pensamiento, el hombre no solo busca la unidad. La tarea del espíritu no es solo reducir lo múltiple a lo uno. Por el contrario, nuestras facultades de análisis deben multiplicar lo más posible los aspectos del universo, y la verdadera unidad debe buscarse más alto, en una intuición que sería una concentración sobre todo más densa de puntos de vista que el análisis ha conducido más lejos. ${ }^{62}$

Se trata de contemplar los grados del ser donde se realiza con más o menos perfección una concentración a la vez densa y coloreada. Y ¿se puede ir aún más lejos? Es decir ¿hay también no-Ser? Hacia el final de la memoria aparece entonces el problema del mal que, como no-Ser, es la falta paradójica que paraliza y moviliza la reflexión, de allí su "necesidad" metafísica. Como muestra Ricœur el descubrimiento de esta falta a través del método reflexivo aplicado a la reconstitución histórica del problema de Dios, es un problema insoluble para Lachelier, pues si todo se deriva de Dios ¿cómo explicar que el hombre opte por el mal? El monismo de Lagneau lo transforma en una alternativa moral: ser o no ser. Para el joven Ricœur su paradoja ofrece la posibilidad de un cambio de posición: "Me pregunto si el impersonalismo no es responsable de ello; quizás el mal esté relacionado con nuestra autonomía personal; quizás el mal es solo un fruto de la libertad y una tarea personal. El problema del sufrimiento a menudo se confunde con el problema del mal: el primero es un problema universal, el segundo es un problema humano." ${ }^{63}$ Nuevos "quizás" indican una prolongación de la metafísica que le obliga a integrar el problema moral del mal porque la moviliza hacia la trascendencia inmanente: "el mal es un problema; pero el mal es el hecho de una persona y un producto de la libertad. [...] Por lo tanto, hay grados de ser, pero no de no ser." ${ }^{4}$ Ante el mal, la metafísica se llegará a convertir bajo la influencia de la filosofía reflexiva de Nabert en una metafísica del testimonio ético de Dios. ${ }^{65}$ Pero eso será otra historia futura. En el inicio de su camino, la metafísica que con su método reflexivo y en su camino genético nos remontaba a Dios, descubre la paradoja que ahora la moviliza a una larga vía ascendente y exterior que se formula en las preguntas y frases bíblicas con las que Ricœur acaba su memoria: “¿Por qué ahora el absoluto no es solamente el

62 Ricœur, Méthode réflexive appliquée au problème de Dieu, 243. Aquí están losantecedentes primeros de lo que luego llamará la función meta- del pensamiento, que será caracterizada por esas dos estrategias complementarias (jerarquización y diversificación), y conjugada con una hermenéutica del sí, regirá la transición que conduce de la metafísica a la moral en una relación dialéctica que las liga y distancia. Cf. Ricœur, "De la métaphysique à la morale", en Autobiografía intelectual, 115. En vistas de la autointerpretación de su camino, que hace en Soi-même comme un autre y que fue citado al inicio de este trabajo, es interesante que en este texto de los años 90, contenido en su Autobiografía, Ricœur se proponga "esbozar los rudimentos de mi propia respuesta, gracias a una reflexión de segundo grado aplicada a aquellos de mis últimos trabajos [en especial, Soi-même comme un autre] que comportan una toma de posición, explícita o implícita, sobre el uso de los términos 'metafísica' y 'moral', y su eventual interdependencia." Ibídem, 87.

63 Ricœur, Méthode réflexive appliquée au problème de Dieu, 243.

64 Ricœur, Méthode réflexive appliquée au problème de Dieu, 244.

65 A partir de la influencia de la filosofía reflexiva de Jean Nabert, la cuestión metafísica de Dios no se podrá separar en Ricœur de la noción ética de testimonio que lo recepciona y sugiere la idea de un absoluto personal cuya afirmación absoluta por la mediación de ese testimonio consiste en un despojo (dépouillement) a la vez ético y especulativo. Cf. Paul Ricœur "L’herméneutique du témoignage”, en E. Castelli (éd.), Le témoignage (Paris: 1972), 36 y ss. Esta respuesta influirá en el injerto hermenéutico en el programa de su Filosofía de la Voluntad bajo los aportes de Nabert al problema del mal. 
punto? ¿Cómo entender este movimiento que lleva de Dios al mundo? En todo caso, es concebible que, en la perspectiva de la trascendencia, eso sea la prerrogativa de Dios en tanto que distinto de nuestra reflexión. Los caminos de Dios no son nuestros."66

\section{Concentración final de una conclusión imposible}

Si he llegado a entender algo razonable, creo que la idea de metafísica y la cuestión de Dios -el de los filósofos y el Otro-, a las que Ricœur se refiere en su memoria no remiten a una metafísica como síntesis onto-teológica. Refiere a lo que llamaría la intuición de un esfuerzo integral inherente a la labor filosófica: 1) de comprender, a través del método reflexivo, el ser como voluntad y libertad; y 2) de reflexionar, actuar y recibir, a través del método prospectivo, esa comunión que es culminación de la tendencia latente en la totalidad de los seres. De esta petite métaphysique no juzgo ni su factibilidad práctica, ni su grado de coincidencia con la metafísica y la cuestión filosófica de Dios, de las que Ricœur quiere distanciarse en su autointerpretación madura, ni la posibilidad de ser la razón causal de la dualidad de su camino entre obras filosóficas agnósticas y obras de exégesis bíblica. Si hubiera que resumirla, diría que es: a) una metafísica finita donde Dios obliga al inacabamiento de una culminación siempre anticipada, pero nunca alcanzada; b) una metafísica nacida del método reflexivo que es necesariamente regresiva porque en el camino genético se topa con la trascendencia inmanente a la diversidad de los grados del ser, y necesariamente proyectiva porque su reflexión en acción es recepción -como acto personal en la estructura impersonal del pensamiento- de esa diversidad que da paso a la vida personal moral y religiosa; c) finalmente, una metafísica de la trascendencia en la inmanencia, donde la distancia y la ausencia, que no puede sintetizar, la obligan a prolongarse por largos rodeos extraños, convirtiéndola en una metafísica del esfuerzo o en un esfuerzo metafísico y moral que debe dar lugar a nuevas obras siempre inacabadas.

\section{Bibliografía}

Blondel, Maurice. "Le point de départ de la recherche philosophique". Annales de philosophie chrétienne, (1906): 234-235. Dosse, François. Paul Ricœur. Los sentidos de una vida (1913-2005). Buenos Aires: FCE, 2008.

Dosse, François. Paul Ricour: Les sens d'une vie (1913-2005). Paris: La Découverte/ Poche, 2008.

Greisch, Jean. "Préface". Méthode réflexive appliquée au problème de Dieu chez Lachelier et Lagneau, (Philosophie \& théologie). Paris: Editions du Cerf. Edición de Kindle, 2017.

Grondin, Jean. “¿En qué consiste el sentido hermenéutico?”. VII Seminario de Fenomenología existencia: Sensibilidad y hermenéutica tras una ética de la solicitud. Temuco: Universidad de la Frontera, 2018.

Grondin, Jean. "Paul Ricoeur, Méthode réflexive appliquée au problème de Dieu chez Lachelier et Lagneau”. Revue des sciences religieuses 92, no. 1 (2018): 121-127. http://journals.openedition.org/rsr/4409, p. 2.

Grondin, Jean. "De la dimension métaphysique de l'herméneutique". Du sens des choses. L'idée de la métaphysique. Paris: Presses Universitaires de France, "Chaire Etienne Gilson", 2013. https://www.cairn.info/du-sens-deschoses--9782130625643-page-143.htm

Lachelier, Jules. "Sur le problème religieux et la dualité de la nature humaine". Société française de philosophie 2 (1913): 167-171.

66 Ricœur, Méthode réflexive appliquée au problème de Dieu, 244. La frase bíblica es "Los caminos de Dios no son nuestros", Isaías 55, 8. 
LaCocque, André y Paul Ricoeur. "Pensar la Biblia”. Estudios exegéticos y hermenéuticos. Barcelona: Herder, 2001. Lagneau, Jules. De la existence de Dieu. Paris: Félix Alcan, 1925.

Ricœur, Paul. "L’herméneutique du témoignage". En E. Castelli (éd.), Le témoignage. Paris: 1972.

Ricœur, Paul. "La hermeneútica bíblica”. Edicoes Loyola, 2001.

Ricœur, Paul. Autobiografía intelectual. Buenos Aires: Ed. Nueva Visión, 2007.

Ricœur, Paul. Exégesis y hermenéutica. Madrid: Cristiandad, 1971.

Ricœur, Paul. Hermenéutica de la idea de Revelación. 1977.

Ricœur, Paul. Hermenéutica del testimonio. Texto, testimonio y narración, 1972.

Ricœur, Paul. La filosofía de la especificidad del lenguaje religioso. 1975.

Ricœur, Paul. La memoire, l'histoire, l'oubli. Paris: Le seuil, 2000.

Ricœur, Paul. Lectures III, Aux frontières de la philosophie. Paris: Ed. Le Seuil, 1994.

Ricœur, Paul. Manifestación y proclama. 1974.

Ricœur, Paul. Nombrar a Dios. 1977.

Ricœur, Paul. Philosophie de la volonté I. Paris: Aubier, 1950.

Ricour, Paul. Soi-même comme un autre. Paris: Ed. Du Seuil, 1990.

Ricœur, Paul. Vivant jusquả la mort, suivi de Fragments: Paris: Seuil, 2007.

Vallée, M-A. "Le premier écrit philosophique de Paul Ricœur". Méthode réflexive appliquée au problème de Dieu chez Lachelier et Lagneau", Études Ricœur iennes / Ricœur Studies, Vol 3, No 1 (2012).

Vieillard-Baron, Jean-Louis. "Les origines spiritualistes de la pensée de Paul Ricœur". Revue des sciences religieuses, 92, no. 2 (2018): 245-262. 